\title{
Minimally Invasive Direct Coronary Artery Bypass for the management of Anomalous Left Coronary Artery from the Right Coronary Sinus
}

\author{
Amit Pawale, MD, Mitsuko Takahashi, DO, Karthik Seetharam, MD, Ramachandra C. Reddy, MD \\ Mount Sinai Medical Center, Department of Cardiothoracic Surgery, New York, New York
}

\section{ABSTRACT}

Objective: Anomalous left coronary artery from the right coronary sinus (ALCA) is a known cause of sudden death. Surgical correction is recommended for all patients with interarterial course. We describe two patients who underwent surgical correction through an off pump- minimally invasive direct coronary artery bypass (MIDCAB) approach with good short- and mid-term results.

\section{INTRODUCTION}

Anomalous origin of the left coronary artery from the right coronary sinus (ALCA) can cause sudden cardiac death [Taylor 1992]. Surgery is recommended for all patients with this diagnosis and a malignant interarterial course [Romp 2003]. Surgical options include coronary artery bypass grafting (CABG), un-roofing of the intramural portion of the aberrant artery and re-implantation of the ostium of the left coronary artery into the appropriate aortic sinus [Romp 2003; Davies 2009]. In patients undergoing conventional CABG (midline sternotomy with cardio-pulmonary bypass (CPB)) left internal mammary artery (LIMA) to the left anterior descending artery (LAD) anastomosis has been the standard approach [Cohen 1989]. More recently, a minimally invasive direct approach for single vessel LAD bypass through a left anterior thoracotomy (MIDCAB) without the use of CPB has been described for the treatment of atherosclerotic coronary artery disease (CAD) [Reddy 2012]. We describe the use of this approach in two patients with ALCA with good shortand mid-term results. To our knowledge this approach to ALCA has not previously been reported.

\section{METHODS}

Mount Sinai Medical Center in New York participates in the New York State Department of Health (NYSDOH) electronic database, which includes all patients undergoing cardiac surgery at the institution. A retrospective review of patients undergoing cardiac surgery revealed 41 patients who underwent surgery for anomalous coronary origin from the

Received Fanuary 10, 2017; accepted April 26, 2018.

Correspondence: Ramachandra Reddy, Mount Sinai Medical Center, Department of Cardiothoracic Surgery, 1190 5th Avenue, Box 1028, New York, NY 10029; 212241-9323; fax: 212-241-0851 (e-mail: ramachandra.reddy@mountsinai.org). opposite sinus of Valsalva between March 2008 and April 2016. Sixteen of these patients had ALCA. We describe two adult patients with ALCA who were operated upon using a MIDCAB technique. Data collection and management of patient information was conducted according to institutional review board guidelines. Additional data was collected through medical chart review and individual consent was waived due to the retrospective nature of data collection. Exposure of individually identifiable personal health information was prohibited.

\section{PATIENTS}

Case 1:A 46-year-old male with a history of hypertension and hyperlipidemia who complained of progressively worsening exertional dyspnea and chest pain for two years exacerbated three weeks prior to admission. Nuclear stress testing revealed anterior wall ischemia and cardiac catheterization showed an anomalous origin of the left main coronary artery from the right coronary sinus without obstructive coronary artery disease (Figure 1). CT angiography confirmed ALCA and demonstrated an interarterial course between the aorta and pulmonary artery (Figure 2) with muscle bridging and compression.

Case 2: A 62-year-old male with a past medical history significant for hypertension, hyperlipidemia, Type 2 diabetes,

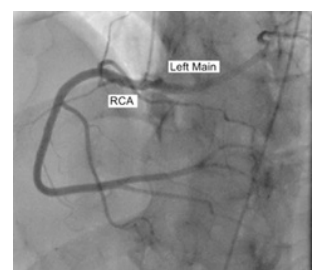

Figure 1. Coronary angiography of anomalous left coronary artery.

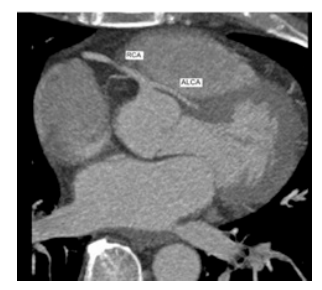

Figure 2. CT Angiogram of anomalous left coronary artery. 


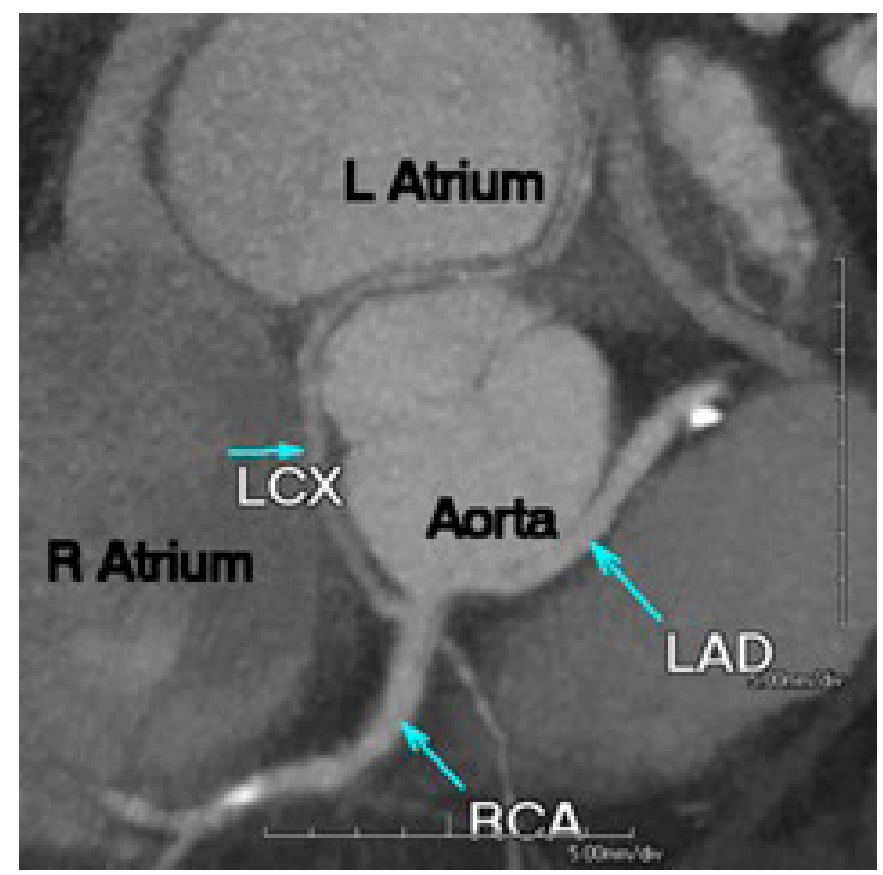

Figure 3. CT Angiogram of anomalous origin of the LAD from the right coronary sinus and anomalous origin of the Circumflex from the RCA.

and renal insufficiency who presented to the hospital for chest pain, exertional dyspnea and fatigue for the past six months. Stress testing revealed anterolateral ischemia and coronary angiography revealed an anomalous origin of the left coronary artery from the right coronary sinus with an interarterial course and an additional anomalous origin of a circumflex artery from the proximal right coronary artery with a retroaortic course (Figure 3 ).

\section{TECHNIQUE}

Intraoperatively, the patients were intubated with a double lumen endotracheal tube and defibrillator pads were carefully placed. They were positioned with the left side slightly up and a $5 \mathrm{~cm}$ to $7 \mathrm{~cm}$ sub-mammary incision was made. The chest was entered through the fifth intercostal space. The LIMA was identified and carefully harvested under direct vision to the level of the subclavian vein using a skeletonized technique. After heparinization with a goal ACT of $>300$ seconds, the LIMA was divided distally. The pericardium was opened anterior to the phrenic nerve and the LAD identified and stabilized using a compression stabilizer (MAQUET Cardiovascular, Wayne, NJ). The proximal LAD was occluded using a silastic loop and a longitudinal arteriotomy was performed. An appropriately sized intra-coronary shunt was introduced and the LIMA to LAD anastomosis created in an end to side fashion using 7-0 prolene. The shunt was removed prior to completion of the anastomosis and Heparin was reversed with Protamine. Transit time ultrasound was utilized to verify flow. The pericardium was loosely reapproximated taking care to avoid any kinking of the LIMA. An intercostal nerve block

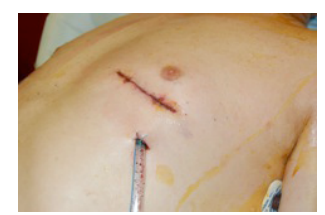

Figure 4. Postoperative appearance of Left MIDCAB incision with single chest tube.

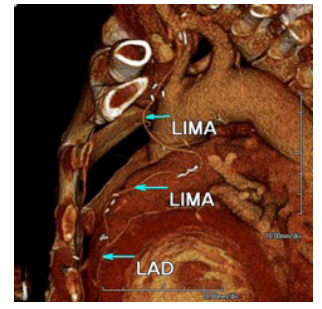

Figure 5. Postoperative CT Angiogram demonstrating a patent LIMALAD graft.

was performed and a single chest tube inserted prior to standard chest closure (Figure 4).

Both patients recovered well and were discharged home on postoperative day four and five, respectively (average 4.5 days). The first patient had a stress exam three months after surgery, which was negative for ischemia and remains asymptomatic 94 months following surgery. The second patient is also asymptomatic 63 months after surgery and had a postoperative CT angiogram demonstrating a patent LIMA-LAD graft (Figure 5).

\section{DISCUSSION}

Myocardial ischemia from ALCA is associated with a variety of factors, including a slit-like ostium, an acute angle of takeoff from the aorta and compression of the intramural portion of the aberrant vessel [Romp 2003]. Surgery is recommended for all patients diagnosed with ALCA with interarterial course [Romp 2003; Davies 2009; Cohen 1989]. There are two contrasting philosophies in surgery. Anatomic corrections include un-roofing the intramural portion of the aberrant artery, reimplantation of the ostium in the appropriate sinus, and enlargement of the slit-like ostium by patch angioplasty. The other approach is by CABG. Unfortunately, each of these options has drawbacks. Un-roofing is only possible if there is good intramural segment and can be associated with aortic valve injury [Romp 2003]. The long-term patency of the neo-ostium created by un-roofing or re-implantation or patch repair is unknown [Romp 2003]. Reimplantation requires extensive mobilization of the proximal left main trunk, which is technically challenging in adults. Also, the anastomosis done without a button can be fragile. Competitive flow between the native coronary artery and the LIMA is an issue under resting conditions, but the large vascular bed of the left coronary system may reduce this risk [Cohen 1989]. Coronary artery bypass grafting with LIMA to LAD bypass continues to be commonly performed for this indication. 
During the last decade, MIDCAB has been performed with increasing frequency at our institution for isolated LAD disease. We have previously reported our series of Right MIDCAB for patients with anomalous right coronary artery origin [Reddy 2012]. The advantage of this technique is that it avoids sternotomy, aortic manipulation, and CPB [Reddy 2012]. In addition, this non-sternotomy approach eliminates the risk of mediastinitis and may be associated with earlier recovery and return to work [Calafiore 1996; Repossini 2000]. A potential disadvantage of this approach is the small incidence of chronic pain associated with thoracotomy. In our opinion, this approach is particularly applicable to the group of patients with ALCA who are often diagnosed at a younger age. Early return to work and cosmesis are important considerations in this group of patients.

A second limitation of MIDCAB in patients with CAD is the identification of an appropriate anastomotic site through a small incision particularly with diffuse LAD disease. ALCA patients have an ostial lesion involving the left main coronary artery with normal distal vessels. Therefore, the identification of an anastomotic site is relatively simple even through a small incision. Another potential technical difficulty with MIDCAB could be insufficient length of the LIMA to reach the anastomotic site. In our cases, adequate mammary length was ensured by using a fifth intercostal space incision, skeletonized harvest technique, and by completing mammary harvest to the level of the subclavian vein.

Early patency of the LIMA in MIDCAB patients has been excellent [Calafiore 1996; Repossini 2000]. Long-term patency for the LIMA to LAD anastomosis in patients with atherosclerotic coronary artery disease is excellent with little late attrition [Jaffrey 2007]. In summary, we present two symptomatic patients with ALCA who underwent a MIDCAB procedure with resolution of symptoms. We believe the
MIDCAB approach is advantageous in patients with ALCA. Short- and mid-term results are favorable, but the long-term outlook is unknown.

\section{REFERENCES}

Barner HD, Barnett M. 1994. Fifteen to 21 year angiographic assessment of internal thoracic artery as a bypass conduit. Ann Thorac Surg 57:1526.

Calafiore AM, Angelini GD, Bergsland J, Salerno TA. 1996. Minimally invasive coronary artery bypass grafting. Ann Thorac Surg 62:1545-8.

Cohen AJ, Grishkin BA, Helsel RA, Head HD. 1989. Surgical therapy in the management of coronary anomalies: Emphasis on utility of internal mammary artery grafts. Ann Thorac Surg 47:630-7.

Davies JE, Burkhart HM, Dearani JA, Suri RM, Phillips SD, Warnes CA et al. 2009. Surgical management of anomalous aortic origin of a coronary artery. Ann Thorac Surg 88;844-8.

Jaffrey A, Kowalski M, Weaver D, Khanal S. 2007. A meta-analysis of randomized control trials comparing minimally invasive direct coronary bypass grafting versus percutaneous coronary intervention for stenosis of the proximal left anterior descending artery. Eur J Cardiothoracic Surg 31:691-7.

Reddy RC, Takahashi M, Beckels DL, Filsoufi F. 2012. Anomalous right coronary artery from the left sinus: A minimally invasive approach. Eur J Cardiothorac Surg 41:287-90.

Repossini A, Moriggia S, Cianci V, Parody O, Sqanzerla P, Bldrighi G et al. 2000. The LAST operation is safe and effective: MIDCAB clinical and angiographic evaluation. Ann Thorac Surg 70:74-8.

Romp RL, Herlong R, Landolfo CK, Sanders SP, Miller CE, Ungerleider RM et al. 2003. Outcome of unroofing procedure for repair of anomalous aortic origin of left or right coronary artery. Ann Thorac Surg 76:589-96

Taylor AJ, Rogan KM, Virmani R. 1992. Sudden cardiac death associated with isolated congenital coronary artery anomalies. J Am Coll Cardiol 20;640-7. 